\title{
A kind of DC fault current-limiter with instantaneous by-pass of current-limiting reactor
}

\author{
Jinyi Deng ${ }^{1}$, Chen $\mathrm{Li}^{2 *}$ and Junfeng $\mathrm{Gao}^{3}$ \\ ${ }^{1}$ International Energy College, Jinan University, Zhuhai, Guangdong, 519070, China \\ ${ }^{2}$ International Energy College, Jinan University, Zhuhai, Guangdong, 519070, China \\ ${ }^{3}$ Chang yuan Electric Power Co. Ltd, Xining, Qinghai, 810021, China
}

\begin{abstract}
With the rapid development of DC power grids, the protection of DC power grids is particularly important. Therefore, this paper proposes a kind of DC fault current-limiter with instantaneous by-pass of current-limiting reactance, analyses its working principle and theoretical calculations, and verifies the correctness of its principle through simulation experiments. At the same time, the experimental results show that the current-limiter proposed in this paper can not only instantaneously and greatly limit the fault current when a short-circuit fault occurs in the power grid, but not affect the normal operation of the power grid. A new device is provided for DC gird fault current-limiting protection.
\end{abstract}

\section{Introduction}

In recent years, with the global energy and power interconnection and the large-scale application of power electronic devices[1], DC power grid and DC transmission technology have developed rapidly and gradually applied to the market, providing technical support for clean energy access to the grid, and improving energy utilization rate to achieve sustainable development.

Compared with traditional AC power grids, DC power grids have the characteristics of low damping, low inertia and slow response time, resulting in poor system tolerance to short-circuit faults [2]. At this stage, the DC grid protection mainly adopts the technical means that the DC current limiter and the circuit breaker cooperate to provide conditions for the operation of the circuit breaker by suppressing the rise of the fault current. The existing superconducting current limiters have problems such as high cost, large size, technical difficulty, solidstate current limiters require many components, and large on-state losses. In addition, when a fault occurs, both types of current limiters cannot instantaneously limit current, and are affected by the main circuit switch.

This paper proposes a DC fault current limiter for instantaneous bypass of current-limiting reactance. It has the following advantages:

- It uses a small number of power electronic devices, low cost, small size, and easy to install.

- When a tidal flow occurs, the current limiter can quickly bypass and reconnect to the grid after rapid stabilization, without affecting the normal operation of the grid.

\footnotetext{
Corresponding author's e-mail: 1c9994@stu2018.jnu.edu.cn
}

- When a fault occurs, the current can be instantaneously limited to improve the efficiency of protection.

- When the fault current is cleared, the current limiter quickly bypasses, which can reduce the disconnection time of the fault current.

\section{The topology of current-limiter}

The topology of the current limiter proposed in this paper is shown in Figure 1. The current limiter is mainly composed of two parts, the current limiting branch and the converter branch. The converter branch routing diodes D1, D2, D3, D4 and IGBT3 form H-bridges, so that the current-limiting reactor $\mathrm{L}$ can bypass or connect to the DC line in different operating states of the power grid. The current-limiting branch is composed of a current-limiting reactor $\mathrm{L}, \mathrm{H} 1$ module and $\mathrm{H} 2$ module. H1 module is composed of diode D5, IGBT1 and lightning arrester, H2 module is composed of diode D6, IGBT2, DC power supply and resistance.

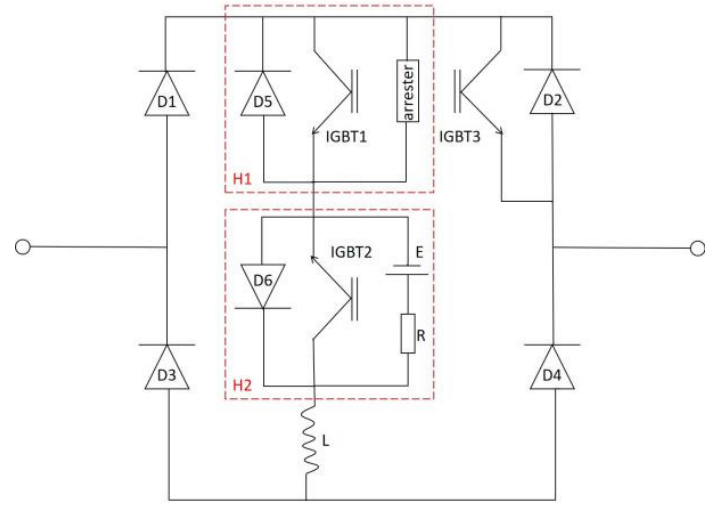

Figure 1. The topology of current-limiter 


\section{The working principle of current limiter}

The current limiter mentioned in this paper has four operating states, namely, normal operation state, power flow moving operation state, fault current limit state, fault current clear state.

\subsection{Normal operation state}

Give IGBT1 and IGBT2 turn-on pulses and IGBT3 turnoff pulses. At this time, the current Idc flows through D1, D4 and the current-limiting branch, as shown in Figure 2. In this state, the lightning arrester that consumes the energy storage of the current-limiting reactor in $\mathrm{H} 1$ and $\mathrm{H} 2$ and the DC power supply that controls the current increase of the current-limiting reactor are short-circuited by IGBT1 and IGBT2 respectively, which does not affect the normal operation of the DC line.

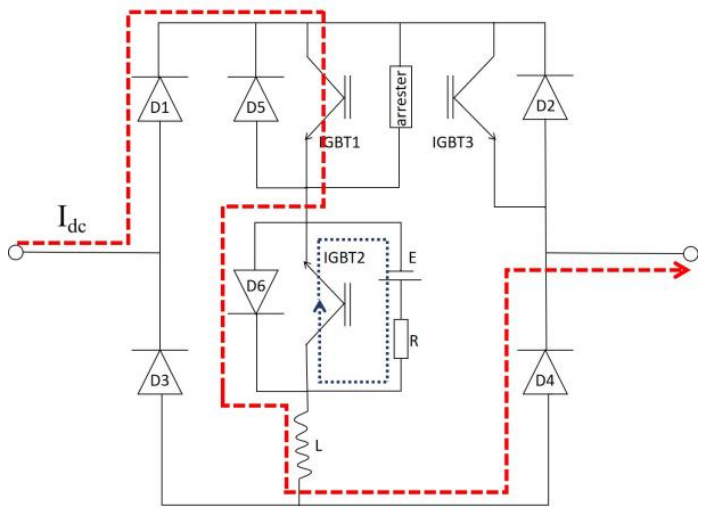

Figure 2. Normal operation state

\subsection{Power flow moving operation state}

In the normal distribution network operation, the movement of the power flow in the line often occurs. If reactance is connected in the line, it will seriously affect the response speed of the line. Therefore, the currentlimiting reactor should be bypassed quickly when the power flow of the line moves, and then the currentlimiting branch current and the line will be stabilized under the action of the $\mathrm{H} 1$ and $\mathrm{H} 2$ modules. The currents are equal. It can be divided into two states: the trend is increasing and decreasing.

\subsubsection{Power flow reduction.}

When the system detects that the power flow decreases, the current Idc decreases instantaneously. After the time T1, the DC circuit breaker does not operate, and the system judges that the power flow decreases. Given the on pulse of IGBT3, the current Idc flows through D1 and IGBT3. The IGBT1 is given a turn-off pulse, and the arrester connected in parallel with it is put into the current-limiting loop, and the energy stored in the current-limiting reactor is consumed by the arrester, as shown in Figure 3. When the current flowing through IGBT3 is close to 0 , IGBT1 is given a turn-on pulse,
IGBT3 is given a turn-off pulse, the current-limiting reactor is reconnected to the line, and the power grid enters a normal operating state.

\subsubsection{Power flow increase.}

When the system detects that the power flow increases, the current Idc increases instantaneously. After the time $\mathrm{T} 1$, the DC circuit breaker does not operate, and the system judges that the power flow is increasing. Given the on pulse of IGBT3, the current Idc flows through D1 and IGBT3. Give IGBT2 a turn-off pulse, and the DC power supply in parallel with it is put into a currentlimiting loop, and the current flowing through the current-limiting reactor begins to increase, as shown in Figure 3 . When the current flowing through IGBT3 is close to 0 , IGBT2 is given a turn-on pulse, IGBT3 is given a turn-off pulse, the current-limiting reactor is reconnected to the line, and the power grid enters a normal operating state.

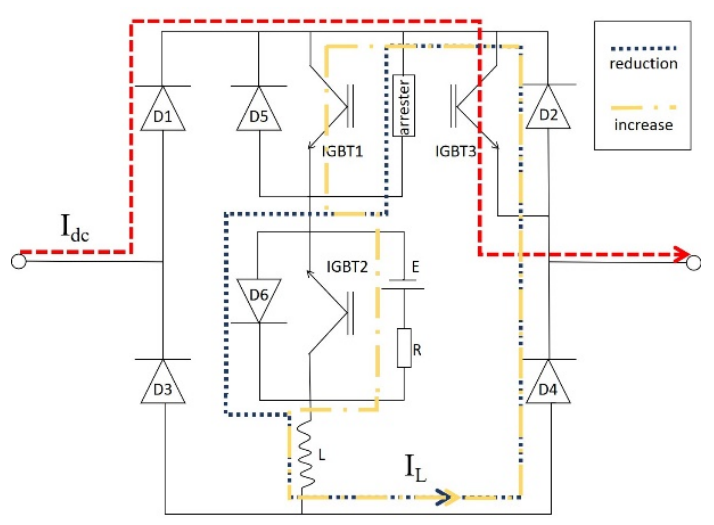

Figure 3. Power flow.

\subsection{Fault current limit state}

When the detector detects an abnormal increase in the line current, the timer is started. If the DC circuit breaker is turned off after the T2 time, it indicates that the DC line current change is caused by the fault. The currentlimiting reactor can have no time delay during the T2 time. Limit the fault current in time and place, effectively limiting the fault current.

When the DC circuit breaker on the fault line is opened instantaneously, the line reactor will automatically bypass, and the current limiter will receive the local DC circuit breaker trip signal after a set time $\triangle \mathrm{t} 1$ (from the start time). Among them, $\triangle \mathrm{t} 1$ should be greater than the tripping time of the DC circuit breaker to ensure that the current change caused by the fault can be reliably determined.

After $\triangle \mathrm{t} 2$, the IGBT1 is turned off, and the lightning arrester connected in parallel with it is put into operation, and the energy stored in the current-limiting inductor is consumed.

When the circuit breaker reclosing is detected, a turnon pulse is given to IGBT1, and the current limiter returns to the current flow state. 


\subsection{Fault current clear state}

Take the ABB hybrid DC circuit breaker as an example. After the main circuit breaker is turned off, the lightning arrester connected in parallel with it starts to consume energy, causing the line current to drop immediately. If the current-limiting reactor is still connected in series in the line at this time, the current-limiting inductance will inhibit the attenuation of the fault current, making the DC circuit breaker disconnect the fault current longer, and the current-limiting reactor stores a lot of energy during the current-limiting process If this part of the energy is consumed by the arrester in the circuit breaker, it will definitely increase the design cost of the arrester. Therefore, it is necessary to realize the fast bypass of current-limiting reactance at the beginning of the operation of the lightning arrester of the DC circuit breaker.

The system detects a line failure, the main circuit breaker of the DC circuit breaker trips, and the arrester starts to consume energy. At the beginning of the main circuit breaker trip due to the presence of IGBT3, the current-limiting reactor is bypassed by IGBT3. In order to ensure that the lightning arrester in the DC circuit breaker has completed the energy consumption in the line, IGBT1 will be given a turn-off pulse after $\Delta t 2$, and the parallel lightning arrester will be used Work starts to consume the energy stored in the current-limiting reactor. Among them, $\Delta \mathrm{t} 2$ should be greater than the DC circuit breaker fault current clearing time.

\section{Theoretical analysis of the current limiting process of current-limiter}

This section conducts theoretical analysis on the fault current limit state. Assume that the distribution network operating system is shown in Figure 4, where Rs is the internal resistance of the DC power supply, Ldc is the smoothing reactor, Rline and Lline are the equivalent impedance of the line, and $\mathrm{R}$ is the load. Regardless of the dynamic characteristics of the capacitor discharge in the converter station, the DC source Udc is used instead [3-4]. The reference direction of each physical quantity is shown in the figure.

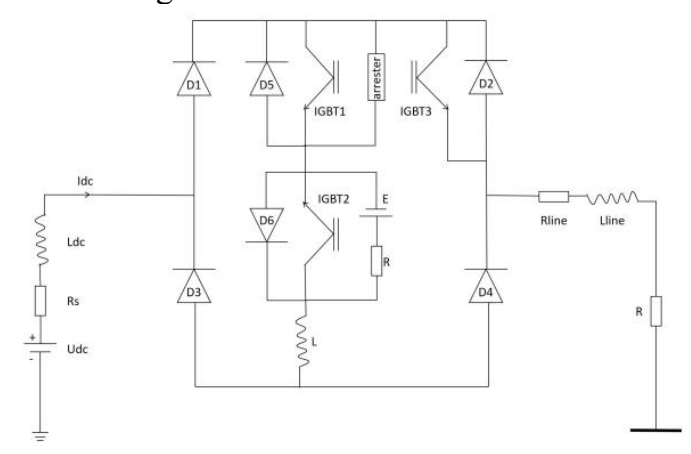

Figure 4. The distribution network operating system.

- Suppose the system detects an abnormal change in line current at $\mathrm{T} 0$, starts a timer, and after $\Delta \mathrm{t} 1$, the current limiter receives a DC circuit breaker opening signal, and the system determines that the current change is caused by a line fault.

$$
\begin{gathered}
\text { Initial state: } I_{d c}\left(0_{+}\right)=I_{d c}\left(0_{-}\right)=\frac{U_{D C}}{R_{S}+R_{\text {line }}+R} \\
\text { Steady state: } I_{d c}(\infty)=0 \\
\mathrm{~T}_{0}+\Delta \mathrm{t}_{1}: i_{d c}(t)=I_{d c}\left(0_{+}\right) e^{-\frac{t}{\tau_{2}}} \quad T_{0} \leq t \leq T_{1} \\
\tau_{2}=\frac{L_{d c}+L_{\text {line }+L}}{R_{S}+R_{\text {line }}} \\
T_{1}=T_{0}+\Delta t_{1}
\end{gathered}
$$

- $\quad$ At T1, the main circuit breaker in the DC circuit breaker has been completely disconnected, and the lightning arrester connected in parallel with it enters into operation, and the line current begins to decrease. At this time, the current-limiting reactor is similar to the state of the flow reduction. Because the current-limiting inductance hinders the current reduction, under the action of the forward voltage, the current-limiting reactor $\mathrm{L}$ forms a loop with D4, D2, IGBT1, and D6, and the loop current remains at Idc $(0+)$, in fact, the current-limiting reactance $\mathrm{L}$ is short-circuited by $\mathrm{D} 4$ and $\mathrm{D} 2$.

$$
\begin{aligned}
& \text { Initial state: } I_{d c}\left(0_{+}\right)=I_{d c}\left(0_{-}\right)=i_{d c}\left(t_{1}\right) \\
& \text { Steady state: } I_{d c}(\infty)=0 \\
& \begin{aligned}
\mathrm{T}_{1}+\Delta \mathrm{t}_{2}: i_{d c}(t) & =i_{d c}\left(t_{1}\right) e^{-\frac{t}{\tau_{d c c b}}} \quad T_{1} \leq t \leq T_{2} \\
\tau_{2} & =\frac{L_{d c}+L_{\text {line }}}{R_{S}+R_{\text {line }}+R_{d c c b}} \\
T_{2} & =T_{1}+\Delta t_{2}
\end{aligned}
\end{aligned}
$$

Among them, $\triangle \mathrm{t} 2$ should be greater than the time from the start of the DC circuit breaker arrester to reduce the line current to 0 to ensure that the energy in the current-limiting reactor does not pass through the DC circuit breaker arrester.

\section{Parameter design and experiment}

This section mainly conducts simulation experiments on the two states of power flow movement and fault current limiting to verify the working effect of the current limiter and the correctness of the theoretical analysis. The simulation experiment system is shown in Figure 5. The DC cable line in the system adopts the lumped parameter equivalent, which is equivalent to a series circuit of resistance and inductance, and $\mathrm{R}=0.078 \Omega / \mathrm{km}$, $\mathrm{L}=0.48 \mathrm{mH} / \mathrm{km}[5]$. Assuming the system cable length is $9 \mathrm{~km}$, then Rline $=0.702 \Omega$, Lline $=4.32 \mathrm{mH}$, rated $\mathrm{DC}$ voltage $\mathrm{Udc}=750 \mathrm{~V}$, and current limiting inductance $\mathrm{L}=0.02 \mathrm{H}$.

\subsection{Power flow moving}

- As shown in Figure 5, when $\mathrm{T}=0.3 \mathrm{~s}$, the switch is closed, the resistance in parallel with it is short-circuited, the line resistance decreases, and the current gradually increases. After about 46.638 us, the DC circuit breaker is not disconnected, and the system judges that the power flow is increasing.

- At this time, if the IGBT3 is given a turn-on pulse, the line current Idc flows through D1 and IGBT3 and gradually increases, and the current-limiting reactor is bypassed. The IGBT2 is given a turn-off pulse, and the parallel DC power supply is put into the current-limiting branch, and the current of the current-limiting branch 
gradually increases. After $0.985 \mathrm{~ms}$, the system enters a stable operating state.

- If the current-limiting branch has no bypass, due to the existence of the current-limiting reactor, the time for the line to enter normal operation will increase. After $8.429 \mathrm{~ms}$, the system entered a stable operating state.

- When the power flow increases, the currentlimiting reactor is not bypassed in time. Compared with the timely bypass, the time required for the system to enter a stable operation state is delayed by $7.444 \mathrm{~ms}$.

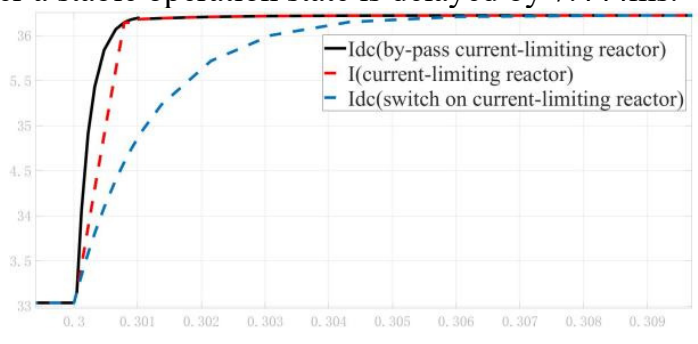

Figure 5. Power flow increase.

In summary, if the current-limiting reactor is not bypassed in time when the system undergoes a tidal flow, it will greatly increase the time for the system to resume normal operation and cause serious energy loss. In addition, the energy stored in the current-limiting reactor needs to be consumed by the arrester in the DC circuit breaker, which increases the design cost of the arrester.

\subsection{Fault current limit}

As shown in Figure 6, when $\mathrm{T}=0.4 \mathrm{~s}$, the switch is closed, and the load connected in parallel with it is shortcircuited, the line has a short-circuit fault, and the current increases instantly. After about $30.293 \mathrm{~ms}$, the DC circuit breaker was disconnected, and the system judged it as a short-circuit fault. From the moment a short circuit occurs to when the circuit breaker is turned off, the current-limiting reactor has been in a current-limiting state, and the current is limited to $635.3 \mathrm{~A}$. When the infinite current reactor is current limiting, the line current will increase to $1.054 \mathrm{kA}$, and the available fault current will be reduced by $39.7 \%$. It can also be obtained from the image that the fault current is reduced by $71.2 \%$ within $5 \mathrm{~ms}$ of the fault.

When the DC circuit breaker is opened, IGBT3 is given a turn-on pulse and IGBT1 is a turn-off pulse, and the lightning arrester connected in parallel with it starts to consume the energy of the current-limiting reactor. After about $110 \mathrm{~ms}$, the energy of the reactor is exhausted.

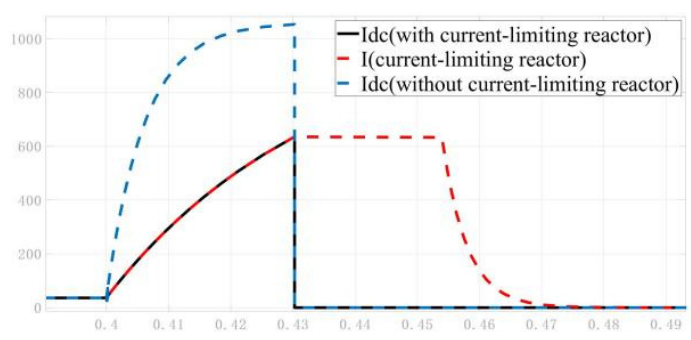

Figure 6. Fault current limit

\section{Conclusion}

This paper proposes a power grid DC fault current limiter with instantaneous bypass of current-limiting reactance, studies its working principle in five power grid operating states, theoretically analyzes the fault current-limiting state, and verifies it through simulation experiments. The conclusions of the current limiter in this paper are as follows:

- Fast bypass does not affect the normal power flow movement of the power grid and the time required for the arrester in the circuit breaker to clear the fault current.

- When connected to the power grid, the fault current is quickly and efficiently limited to ensure the normal operation of the circuit breaker.

\section{References}

1. Zhao Xibei, Xu Jianzhong, Yuan Jinsha, Zhao Chengyong. A new type of capacitor-commutated hybrid DC current limiter[J]. Proceedings of the Chinese Society of Electrical Engineering, 2018, 38(23): 6915-6923+7125.

2. Zhu Sicheng, Zhao Chengyong, Li Chengyu, Xu Jianzhong. Calculation method of DC grid fault current with action of DC fault current-limiting device[J]. Proceedings of the Chinese Society of Electrical Engineering, 2019, 39(02): 469-478+644.

3. Han Naizheng, Jia Xiufang, Zhao Xibei, et al. A new type of hybrid DC fault current limiter topology[J]. Proceedings of the Chinese Society of Electrical Engineering, 2019, 39(6): 1647-1658, 1861.

4. Zhou Meng, Zuo Wenping, Lin Weixing, et al. Capacitor commutation type DC circuit breaker and its application in DC grid [J]. Proceedings of the Chinese Society for Electrical Engineering, 2017, 37(4): 1045-1052.

5. Gao Feng. Research on Relay Protection Principle of DC Distribution System with Resistive Superconducting Current Limiter [D]. Tianjin University, 2016. 